This niport was prepared as an account of work sponsored by an agency of the United States Govermment. Neither the United States Government nor any agency thereuf, nor any of their employees, makes any warranty, express or implied, or assumes any legal liability or reaponsibility for the accuracy, completeness, or usefulness of any information, apparatus, product, or process disclosed, or represents that its use would not infringe privately owned rights. Reference herein to any specific commercial product, process, or service by trade name, trademark, manufacturer, or otherwise does not nccessarily constitute or imply its endorsement, necommendation, or favoring by the United States Government or any agency thereof. The views and opinions of authors expressed herein do not recessarily state or reflect those of the United States Government or any agency thereof.

\title{
DYNAMICS OF ANGULAR MOMENTUM ACCUMULATION IN DAMPED NUCLEAR REACTIONS*
}

\author{
Jorgen Randrup and Thomas Dossing ${ }^{+}$ \\ Nuc lear Science Division, Lawrence Berkeley Laboratory, \\ University of California, Berkeley, California 94720
}

The dynamical evolution of the correlated angular momentum distribution in a damped nuclear reaction is discussed within the framework of the nuc leon exchange transport model.

\section{INTRODUCTION}

The accumulation of angular momentum in a damped nuclear reaction is an important topic for our understanding of low-energy nuclear dynamics. The fragment spins carry considerable information about the reaction dynamics: white the mass and charge distribution can be characterized by two mean values and three covariances, the correlated spin distribution requires two mean values and thirteen non-trivial covarfances. On the other hand, the spin-related observables are more difficult to extrect experimentally.

The present contribution reports briefly on some selected results of a recent study of the dynamical evolution of angular momentum in damped nuclear reactions. ${ }^{1,2}$ The study is carried out within the framework of the nucleon exchange transport model, ${ }^{3}$ in which the dissipation of the macroscopic variables is caused by the inelastic interactions of individual nucleons with the timedependent mean field. In the case of a binary system, as is temporarily created during a damped reaction, this one-body mechanism appears as a "window" dissipation caused by the transfer of nucleons between the two reaction partners, in addition to a "wall" friction caused by the reflection of nueleons from the changing potential in the interaction zone between the two nucleides.

The reacting system is idealized as two spherical nucleides $A$ and $B$. The relative orbital angular momentum is $\vec{L}=\vec{R} \times \vec{P}$ where $\vec{R}$ is the relative position and $\vec{p}$ is the relative momentum. The associated moment of inertia is $y_{R}=4 R^{2}$. The angular monenta, or spins, of the individual nucleides are $\mathcal{S}^{A}$ and ${ }^{B} B$, and $J_{A}$ and $J_{B}$ are the associated moments of inertia. Specific details about this model can be found in appendix $A$ of ref ${ }^{4}$.

*This nork was supported by the Director, Office of Energy Researth, Division of Nuclear Physics of the Office of High Energy and Nuclear Physics of the $J . S$. Department of Erergy under Contract DE-ACO3-76SF00093.

thels Somr Fellow, granted by the Royal Danish Acaremy of Science. 
2. EQUATIONS OF MOTION

in ref. ${ }^{3}$, the mobility tensors relating to the two fragment spins $\vec{S}^{A}$ and $\vec{S}^{B}$ were round to be

$$
\begin{aligned}
& \vec{M}^{A A}=m N\left(a^{2} \stackrel{T}{T}+c_{\text {ave }}^{2} \stackrel{\leftrightarrow}{I}\right) \\
& \vec{M}^{A B}=m N\left(a b \overleftrightarrow{T}-c_{\text {ave }}^{2} \overleftrightarrow{I}\right)=\overleftrightarrow{M^{B A}} \\
& M^{B B}=m N\left(b^{2} \overleftrightarrow{T}-c_{\text {ave }}^{2} \overleftrightarrow{I}\right)
\end{aligned}
$$

Here $\vec{I}$ is the identity tensor and $\vec{T}=\vec{I}-\hat{R} \hat{R}$ projects onto the plane perpendicular to the dinuclear axis $R$. The distances to the "window" plane from the two nuclear centers are denoted by $a$ and $b$, with $a+b=R$, while $c_{a v e}$ is the average off-axis displacement of the transferred nucleons. The nucleon mass is denoted $m$, and $N$ is the overall form factor governing the rate of nucleon transfer between the two nucleides $A$ and $B$.

In addition to the fragment spins $\vec{S}^{A}$ and $\vec{S}^{B}$, it is also necessary to consider the evolution of the orbital angu.ar momentum $\vec{L}$. This is because we wish to use a coordinate system whose direction fluctuates with respect to an external inertial system (and hence the components of the total angular momentum $\vec{j}$ will fluctuate). It -is notationally convenient to denote any of the angularmomentum labels $A, B, L$ by the letters $F, G, \ldots$ so that $\vec{S}^{F}=\vec{S}^{A}, \vec{S}^{B}, \vec{L}$ for $F=$ $A, B, L$, respectively. The mobility tensor relating to the orbital angular momentum can then be obtained by exploiting the conservation of the total angular momentum $\vec{j}=\vec{s}^{A}+\vec{s}^{B}+\vec{L}$.

In terms of the mobility coefficients the spin transport coefficients are given as follows. The diffusion coefficients are simp:y the corresponding mobility coefficients multiplied by the "effective temperature" $T^{*}: D^{F G}=M^{F G}$. The drift coefficients are obtained by multiplying the mobility tensor with the corresponding generalized forces, $i . e .$, minus the rotational frequencies $\vec{w}=$

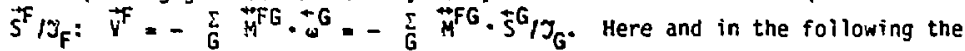
sum over the labeis $G$ extends over $G=A, B, L$.

In order to take full account of the so called tilting mode, it is necessary to employ a "body-aligned" orthonormal reference system. Specifically, we define the coordinate system $x y z: \hat{z}=\hat{R}, \hat{y}=\hat{L}, \hat{z}=\hat{y} \times \hat{z}$. The choice of $\hat{z}=\hat{R}$ ensures that the nobility tensors $\vec{M}^{F G}$ are diagonal in the spatial indices. Since $\hat{y}=\hat{i}$, the orbital angular nomentum $\vec{i}$ has only components in the $y$-direction. He need then consicer the temporal evolution of $s_{x}^{A}, s_{y,}^{A}, s_{z}^{A}, s_{x}^{B}, s_{y}^{B}, s_{z}^{B}$, $L_{y}$. In a standard collision experiment, all are initially zero except for $t_{y}$ wich equals the total angular momentum $\mathrm{J}$. It follows fran the symetry of the 
problem that the mean values $\left\langle S_{x}^{F}\right\rangle$ ans $\left\langle S_{z}^{F}\right\rangle$ and also the covariances ${ }_{x y}^{F G}$ and ${ }_{y z}^{F G}$ will remain zero throughout the reaction.

The equations of motion for the non-vanishing mean values and covariances are given by

$$
\begin{aligned}
& \dot{s}_{y}^{F}=\sum_{G}\left(M_{t}^{F G} S_{y}^{G}+\frac{1}{L_{y}} \sigma_{x x}^{F G} M_{t}^{G L}\right) / I_{G}+\frac{\tau^{\star}}{L_{y}}\left(2 M_{t}^{F L}-\frac{s_{y}^{F}}{L_{y}} M_{t}^{L L}\right) \\
& \dot{\sigma}_{x x}^{F H}=2 \tau \star M_{t}^{F H}-\sum_{G}\left(\sigma_{x x}^{F G} M_{t}^{G H}+M_{t}^{F G} \sigma_{x x}^{G H}\right) / J_{G}-\omega_{R}\left(\sigma_{x z}^{F H}+\sigma_{z x}^{F H}\right) \\
& -\frac{S_{y}^{F}}{L_{y}}\left(2 \tau^{\star} M_{t}^{L H}-\sum_{G} M_{t}^{L G} \sigma_{x x}^{G H} / J_{G}\right)-\left(2 \tau * M_{t}^{F L}-\sum_{G} \sigma_{x x}^{F G} M_{t}^{G L} / J_{G}\right) \frac{S_{y}^{H}}{L_{y}} \\
& +2 T * \frac{S_{y}^{F}}{L_{y}} M_{t}^{L L} \frac{S_{y}^{H}}{L_{y}} \\
& \dot{\sigma}_{y y}^{F H}=2 \tau * M_{t}^{F H}-\sum_{G}\left(\sigma_{y y}^{F G} M_{t}^{G H}+M_{t}^{F G} \sigma_{y y}^{G H}\right) / Y_{G} \\
& \dot{\sigma}_{z z}^{F H}=2 \tau * M_{n}^{F H}-\sum_{G}\left(\sigma_{z z}^{F G} M_{n}^{G H}+M_{n}^{F G} \sigma_{z 2}^{G H}\right) / Y_{G}-\omega_{R}\left(\sigma_{x z}^{F H}+\sigma_{z x}^{F H}\right) \\
& \dot{\sigma}_{x z}^{F H}=-\sum_{G}\left(\sigma_{x z}^{F G} M_{n}^{G H}+M_{t}^{F G} \sigma_{x z}^{G H}\right) / y_{G}-\omega_{R}\left(\sigma_{x x}^{F H}-\sigma_{z z}^{F H}\right)+2 \tau^{\star} \frac{S_{y}^{F}}{L_{y}} M_{t}^{L G} \sigma_{x z}^{G H} / y_{G}
\end{aligned}
$$

Here we have omitted the bracket around the mean values of $s_{y}^{F}$ for notational simplicity, since confision can hardly arise.

In the above equation for the mean value the first term is the drift coefficient. In the equations for the covariances, the first term is the diffusion coefficient (which vanishes for the non-diagonal components) while the subsequent term represents the restoring te $m$ acting to saturate the growth of $\sigma$. The terms containing $\omega_{R}$ arise from the orbital rotatior. which continually mixes the in-plane components. The remaining terms contain $\left\langle L_{y}\right\rangle$ in the denominator and arise from the transformation to the fluctuating coordinate system aligned with $\vec{L}$. These terms are derived under the standard assumption that all spin dispersions are small in comparison with $\left\langle L_{y}\right\rangle$. While this is only well satisfied for larger impact parameters, the equations do remain well-behaved for more central collisions (which contribute only a small part of the reaction cross section) and even for head-on reactions the solutions are ccrrect to within 25:. Although these latter terms are of the corrective type, they are essential in ensuring the proper long-time bahavior of the solutions, namely an approach towards sta:istical equilibrium.

In the preceding we have referred the spin moments to a coordindte system cefined in terns of the instantaneous values of $\hat{R}$ and $\hat{L}$. However, the direction $i$ can not te determined in a collision experiment, so it is recessary to 
transform the results to a coordinate system which can be externally defined. In a collision experiment two directions are readily determined: the beam direction $\hat{t}$ and the asymptotic dinuclear direction $\hat{R}(\infty)$. In terms of these two directions we define the following external coordinate system $X Y Z: \hat{Z}=\hat{R}, \hat{Y}=$ $\hat{R} \times \hat{t}, \hat{x}=\hat{y} \times \hat{z}$. Since the internal and the external coordinate systems have the same $z$-axis, the two are related by a rotation around the $z$-axis, $\mathcal{R}_{Z}(\zeta)$. Since the cistribution of the angle $\zeta$ between the directions $L$ and $Y$ is determined by the in-plane spin variances, the transformation from $x y z$ to $X Y Z$ can be made.

The resulting spin distribution corresponds to a definite impact parameter, given by the specified value of the total angular momentum $\mathrm{J}$. The model also yields equations of motion for the kinetic energy loss and its covariance with the spin variables. Therefore, it is possible to obtain an "observable" spin distribution gated by energy loss rather than impact parameter.

\section{EQUILIGRIUM}

In the preceding we have outlined how the dynamical evolution of the dinuclear spins can be calculated. The resuits of such calculations can best be understood in terms of the appropriate equilibrium solutions and the associated relaxation times.

In analogy with the treatment of the two-particle problem, we introduce the following spins and associated moments of inertia,

$$
\begin{array}{ll}
\vec{s}=\vec{s}^{A}+\vec{s}^{B}, & J_{+}=J_{A}+J_{B} \\
\vec{s}^{-}=J_{-}\left(\frac{\vec{s}^{A}}{y_{A}}-\frac{\vec{s}^{B}}{J_{B}}\right), & J_{-}=\frac{J_{A} J_{B}}{J_{A}+J_{B}}
\end{array}
$$

They are analogous to the total and relative motion, respectively. For a given total angular momentum $\mathrm{J}$, and under the standard assumption that ine variances are small compared to $\left\langle L_{y^{2}}\right\rangle^{2}$, it is straightforward (albeit tedious) to demonstrate that the dynamical spin equations (2) have a unique stationary solution given by

$$
\begin{aligned}
& \left\langle L_{y}\right\rangle=\frac{J_{R}}{J_{0}} J, \quad \quad \hat{j} L=\tau J+\frac{J_{R}}{J_{0}} \hat{y} \hat{y} \\
& \left\langle 5_{y}^{+}\right\rangle=\frac{y_{+}}{J_{0}} J-\tau * J_{+} \frac{J_{0}}{J_{R}} \frac{1}{J}, \quad \vec{j}^{*+}=\tau^{*} J_{+} \frac{J_{0}}{J_{R}}(\hat{x} \hat{x}+\hat{z} \hat{z})+\tau^{*} J_{+} \frac{J_{R}}{J_{0}} \hat{y} \hat{y} \\
& \left\langle S_{y}^{-}\right\rangle=0 \quad \text { o }=z_{-}^{*} y_{-}
\end{aligned}
$$

Where we have included terms to the first order in the effective temperature 
$\tau^{\star}$. During the reaction, the moments of the spin distribution will at each instant evolve towards these equilibrium values, which in turn vary in time due to the time dependence of the relative moment of inertia $R$ and the effective temperature $\tau^{\star}$. Below we shall first discuss the stationary solution in terms of a statistical model, and next we shall discuss the time scales for the approach towards equilibrium.

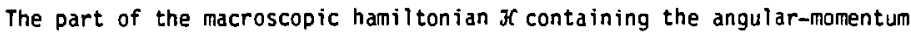
variables in the disphere is

$$
\pi_{\text {rot }}=\frac{\vec{s}^{A 2}}{2 J_{A}}+\frac{\vec{s}^{B 2}}{2 J_{B}}+\frac{t^{2}}{2 J_{R}}
$$

For a given value of $\vec{j}=\vec{S}^{A}+\vec{s}^{B}+\vec{L}$, the lowest-energy mode of rotational motion in the disphere is a rigid rotation with each of the three angular momenta given by $\vec{S}^{F}=J_{F} \vec{J} / J_{0}$ where $J_{0}=J_{A}+J_{B}+J_{R}$. Relative to this yrast mode of motion, intrinsic rotational excitations are possible. These excitations carry no net angular momentum and can be classified in two groups according to whether the two spheres turn in the same or in the opposite sense, i.e., a purely positive mode has $\vec{S}^{-}=\overrightarrow{0}$ and a purely negative mode has $\vec{s}^{+}=\overrightarrow{0}$, where $\vec{S}^{+}$and $\vec{S}^{-}$are given in Eq. (3).

We first consider the problem using the coordinate system $x^{\prime} y^{\prime} z^{\prime}$ defined by $\hat{z}^{\prime}=\hat{R}, \hat{y}^{\prime}=\hat{I}, \hat{x}^{\prime}=\hat{y}^{\prime} \times \hat{z}^{\prime}$, where $\hat{I}=\vec{J}-\hat{J} \times \hat{R} \hat{R}$ is the projection of the total angular momentum $\vec{J}$ on the plane perpendicular to $\vec{R}$. In order to bring the rotational hamiltonian (5) on normal form we introduce the following auxiliary spin variable $\vec{s}=\vec{s}+\left(I_{+} / J_{0}\right) J_{y}, \hat{y}^{\prime}$. This transformation has unit jacobian since $\hat{y}^{\prime}$ is independent of $\vec{S}^{+}$and we obtain

$$
\begin{aligned}
F_{\text {rot }} & =\frac{1}{2 J_{A}}\left(\frac{J_{A}}{J_{+}} \vec{s}^{+}+\vec{s}^{-}\right)^{2}+\frac{1}{2 J_{B}}\left(\frac{J_{B}}{J_{+}} \vec{s}^{+}-\vec{s}^{-}\right)^{2}+\cdot \frac{1}{2 J_{R}}(\vec{J}-\vec{s})^{2} \\
& =\frac{J^{2}}{2 J_{C}}+\frac{1}{2 J_{+}} \frac{J_{0}}{J_{R}}\left(s_{x^{\prime}}^{2}+s_{y^{\prime}}^{2}\right)+\frac{1}{2 J_{+}} \frac{J_{R}}{J_{0}} s_{z^{\prime}}^{2}+\frac{\vec{s}^{-2}}{2 J_{-}}
\end{aligned}
$$

Here the first term represents the yrast energy associated with a rigid rotation while the additional terms arise from the six normal modes of intrinsic rotational excitation of the disphere. The first of these terms is the energy of the two degenerate wriggling modes, where the two spheres rotate in the same sense around an axis perpendicular to $\hat{R}$. The next term is associated with the tilting mode arising when $\vec{J}$ has a component along the dinuclear axis $\hat{R}$; the two spheres thus tura in the same sense around $\hat{R}$. These three are the positive modes. The last term arises fron the three degenerate negative nctes: the ewistisg note, where the two spheres rotate oppositeiy around $\vec{R}$. and the two testisg motes, where the spheres turn cppositely around an ax is 
perpendicular to $\vec{R}$.

Assume now that the rotational modes are weakly coupled to the remainder of the system, which is considered as a heat reservoir with the temperature $\tau$. When $\tau \ll J^{2} / 2$, the six normal rotational modes are approximately harmonic. It is then possible to show that the ensuing themal equilibrium distribution is characterized by

$$
\begin{aligned}
& \left\langle S_{y^{\prime}}^{F}=\frac{J_{F}}{J_{0}} J-\frac{J_{F} I_{+}}{I_{R}} \frac{\tau}{2 J}\right. \\
& \sigma_{y^{\prime} y^{\prime}}^{F G}=\sigma_{x^{\prime} x^{\prime}}^{F G}=\left(\frac{J_{R}}{J_{0}} J_{F} J_{G}+\varepsilon_{F G} J_{A^{\prime}} J_{B}\right) \frac{T}{J_{A}+J_{B}} \\
& \sigma_{Z^{\prime} Z^{\prime}}^{F G}=\left(\frac{J_{0}}{J_{R}} J_{F} J_{G}+{ }_{F G} J_{A} J_{B}\right) \frac{T}{J_{A}+J_{B}}
\end{aligned}
$$

(The symbol $c_{F G}$ is one when $F=G$ and minus one otherwise.) This result is in accordance with the analysis by Moretto. ${ }^{5}$

The above result was expressed in the 1-aligned coordinate system $x^{\prime} y^{\prime} z^{\prime}$. A transformation to our standard "body-fixed" L-aligned system $x y z$ yields the following equilibrium distribution

$$
\begin{aligned}
& \left\langle S_{y}^{F}\right\rangle=\frac{J_{F}}{J_{0}} J-\frac{J_{F} J_{0}}{J_{R}} \cdot \frac{\tau}{J} \\
& \sigma_{Y Y}^{F G}=\left(\frac{J_{R}}{J_{0}} J_{F} J_{G}+c_{F G} J_{A} J_{B}\right) \frac{\tau}{J_{A}+J_{B}} \\
& \sigma_{Z Z}^{F G}=\sigma_{x x}^{F G}=\left(\frac{J_{0}}{J_{R}} J_{C} J_{G}+c_{F G} J_{A} J_{B}\right) \frac{\tau}{J_{A}+J_{B}}
\end{aligned}
$$

This result is identical to the stationary solution (4) of the dynamical equations (2).

In the variances in (7) and (8) the first terns arise from the positive modes (wriggling and tilting) while the second $t$. ns arise from the isotropic negative modes (bending and twisting). The most pronounced effect of the transformation from $x^{2} y^{\prime} z^{\prime}$ to $x y z$ is the increase in the in-plane wriggling variance $a_{x x}^{++}$by the factor $\left(J_{0} / J_{R}\right)^{2}=2$ so that the isotropy in the plane perpendicular to $\vec{R}$ is replaced by isotropy in the plane perpendicular to $\vec{L}$. $A$ difierent nomal form of the rotational hamiltonian (5) for an asymetrical disphere has been introduced by Schmitt and pacheco. ${ }^{6}$ This leads to different definitions of the whighing and bending rodes, but the result expressed in the original variables, eq. (7), is of course the same. 


\section{EVOLUTION IN A SYMMETRIC DISPHERE}

In Section 3 we introduced the spins $\vec{S}^{+}$and $\vec{S}^{-}$; they are particulariy convenient variables when the two spheres are equal. In the symmetric case, where $a=b$ and $J_{A}=J_{B}$, the mixed mobility tensor $\vec{M}^{+-}$vanishes so that the dynamical equations for $\vec{S}$ decouple from the rest; furthermore, the mobility tensor $\mathrm{M}^{-}$is isotropic.

Typical time scales for the approch to equilibrium can be obtained by dividing the asymptotic values by the respective initial time derivatives. This yields for the transversai spin components $\sigma_{x x}^{++}$and $\sigma_{y y}^{++}$the time scales $\frac{J_{0}}{J_{R}} t_{++}$ and $\frac{I_{R}}{I_{0}} t_{++}$, respectively, where

$$
t_{++}=\frac{\tau * J_{+}}{2 \tau * M^{L}}=\frac{J_{+}}{2 \pi N R^{2}}
$$

while for the components of $\ddot{a}^{\not-}$ we find

$$
t_{-}=\frac{\tau * y_{-}}{2 \tau * H-}=\frac{I_{-}}{2 \pi N c_{d v e}^{2}}
$$

Thus, $t_{++} / t \ldots-\left(c_{\text {ave }}^{2} / R^{2}\right) \ll 1$.

Solving the equations more rigorously for the idealized case of constant coefficients, the time, development of the variances are governed by these relaxation times, for example:

$$
\vec{a}--=y_{-} T^{*}\left[1-e^{-t / t}-\right] \vec{I}
$$

The normal variance $\sigma_{22}^{++}$does not receive contributions directly through the transfer process, but only indirectly by the orbital rotation of $a_{x x}^{++}$via ${ }^{++} z^{*}$. Solving the equations for these variances, the relaxation $t$ ime for ${ }^{++} \times x$ is $t_{++}$. which we already discussed, while for $\sigma_{22}^{++}$the typical time scale is

$$
t_{+z}=\left(4_{\omega_{R}^{2}}^{2} \frac{L_{y}}{J_{y}} t_{+}\right)^{-1} \text {, }
$$

which is usually fairly long.

The time scales for the evolution of mean values are approximatelv twice the ones relevant for the variances:

$$
s_{y}^{+}=\frac{I_{+}}{y_{0}}\left[1-e^{-\frac{J_{0}}{I_{R}} \frac{t}{2 t_{++}}}\right], s_{y}^{-}=0
$$




\section{ILLUSTRATIVE RESULTS}

In the preceeding section we have discussed the characteristic features of the spin evolution with an emphasis an the qualitative aspects. We now wish to illustrate the theory quantitatively by making applications to one reaction of actual experimental interest, name 1 y $1400 \mathrm{MeV} 165_{\mathrm{Ho}}+165_{\mathrm{Ho}}$. A pictorial impression of the evolution of the dinuclear geometry can be gained from Fig. 1.

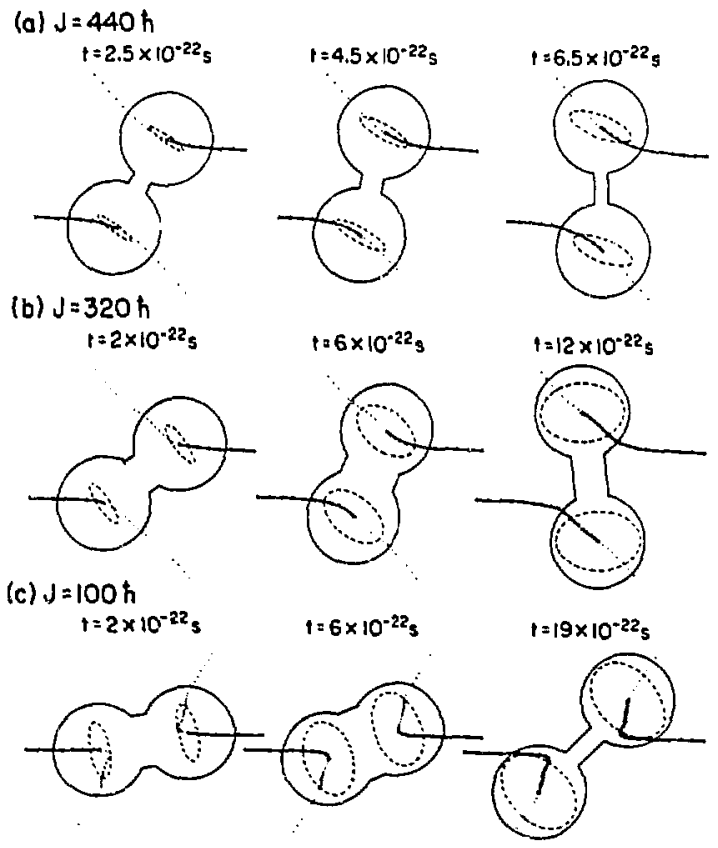

FIGURE 1

For three different values of the total angular moment um $J$, the dinuclear complex produced in the reaction $1400 \mathrm{MeV} 165_{\mathrm{Ha}}+165_{\mathrm{Ho}}$ is shown at three different points in time: shortly after the neck has opened, at the time of closest appradch, and right before the neck collapses. TTe actual tinies indicated are measured from the tine of the nuclei approach to a surface separation of $s=$ $4 \mathrm{fm}$.) The dots indicate past and future locations of the nuclear centers at intervals of $10^{-22} \mathrm{sec}$. The dashed ellipses indic ate the gne-sigma contours of the in-plane distribution of the nuclear engular momenta $J^{A}$ and $\zeta^{B}$ scaled so that one fo corresponds to two (the nuclear radii are $6.3 \mathrm{fm}$ ). 
We now consider in some detail the calculated dynamical evolution of the angular momenta during the reaction phase. First, we consider the various relaxation times introduced in Section 4 . They are shown in Fig. 2 as functions of $t$ ime, for a number of different values of the total angular momentum $\mathrm{J}$. We note that throughout the reaction phase the relaxation times $t_{++}$associated with the two wriggling modes are considerably shorter than $t$ associated with the negative modes, as already expected since $c_{\text {ave }}^{2} \ll R^{2}$. The relaxation time for the tilting mode is fairly long but has an opposite behavior. both as a function of time and in its dependence on J. By comparing the relaxation times with the reaction times it is possible to obtain an expectation for how far the various modes will evolve towards equilibrium. Thus, for not too large impact parameters, we expect the wriggling modes to achieve nearly complete relaxation, contrary to the negative modes for which this is at most expected for the smallest impact parameters. The tilting mode is generally expected to acquire little excitation.

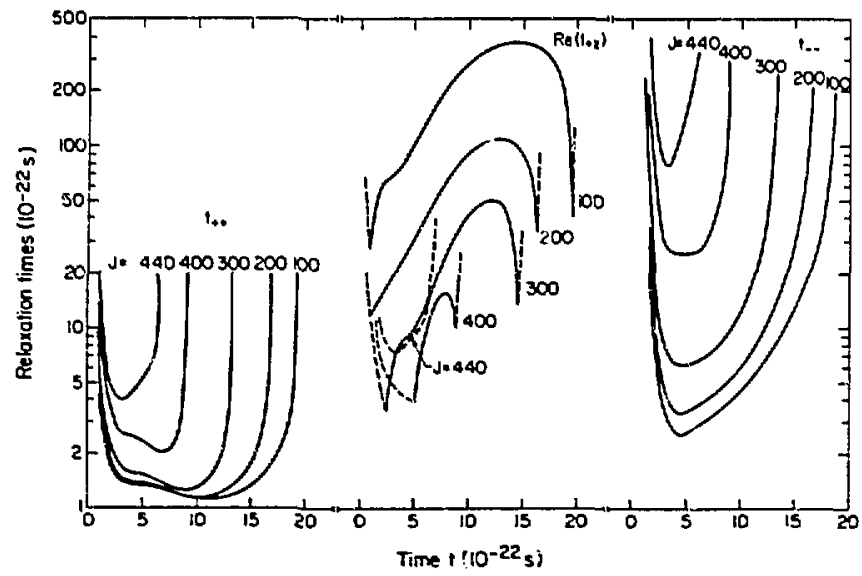

FIGURE 2

Calculated local relaxation times for the reaction $1900 \mathrm{NeV}: 65_{\mathrm{Ho}}+165_{\mathrm{HO}}$ for various values of the total angular comentum 3. The relaxation times for the two positive perpendicular modes (wrigg!ing) are denoted $t+\infty$, while that for the positive longitudinal mode (tiliting) is denoted $t+2$. The relaxation tine for the three negative nodes (bending and twisting) is cenated $t$-.. 
The calculated dynamical evolution of the mean fragment spin projection is shown in Fig, 3, for three selected J-values. For the highest value, $\mathrm{J}=440 \mathrm{~h}$, the reaction is over before the equilibrium mean value can be reached. For the intermediate value, $J=320 \mathrm{~h}$, the equilibrium value is nearly achieved around the time of closest approach. This equilibrium mean spin decreases as the two fragments recede and the relative moment of inertia grows. Therefore, the mean spin exhibits a maximum as a function of time. The same is true at the most central reaction, $J=100 \mathrm{~h}$, but here the equilibrium values are of course smaller.

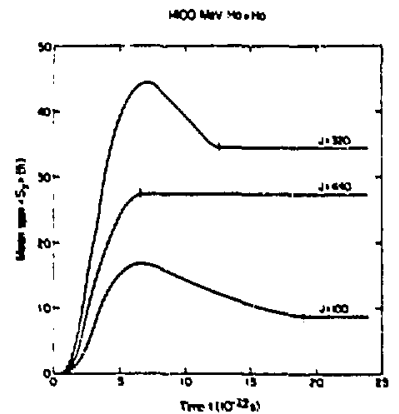

FIGURE 3

Calculated time evolution of the mean fragment spin $\left\langle S_{y}\right\rangle$ in the reaction $1400 \mathrm{MeV} 165$ Ho + $165 \% \mathrm{O}$ for various values of the total angular momentum $\mathrm{J}$. The neck snapping, after which the spins renain constant, is indicated by a small vertical bar.

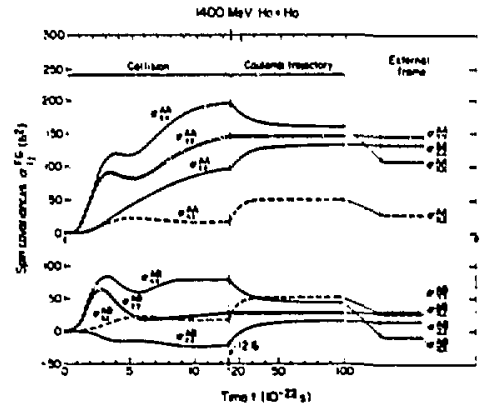

FIGURE 4

Calculated time evolution of the various sp in covariances of $G$ in the reaction 1400 MeV 165 Ho + 165 Ho for a total angular momentum of $\mathrm{J}=320 \mathrm{~h}$. At the time of neck snapping $(t=12.6 \cdot 10-22 \mathrm{~s})$ the time scale is ch.anged by a factor of ten. After the asymptot ic values have been reached, the effect of transfoming to the external reference frame fron $X Y Z$ is shown.

The calculated coin covariances are displayed in $\mathrm{Fig}$. as functions of time. The figure has three parts. The first shows the dyacmical evolution during the reaction phase. It is clearly seen how $0_{x x}^{F G}$ and of $o_{y y}^{F G}$ increase rapidly at early times; this is a refletticn of the fast wriggling rellexation tine [see fig. 2). The local butigs in $o_{x \times}^{F G}$ and $\theta_{y y}^{F G}$ sround the time of

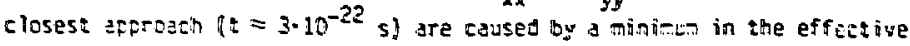

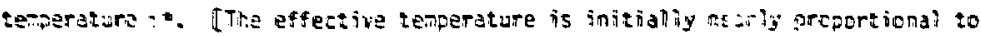


the relative nuclear velocity and hence at first it decreases. Later on, when the relative motion has subsided, $T^{\star}$ is close to the intrinsic temperature $T$ which increases in time. Thus t exhibits a minimum which occurs approximately at the turning point of the relative motion.] The evolution of $\sigma_{z z}^{F G}$ is considerably slower, as expected from Fig. 2. Most of $\sigma_{z Z}^{F G}$ is associated with the negative twisting mode as evidenced by the fact that the covariance $\sigma_{z Z}^{A B}$ is negative, but, as the difference between $\sigma_{2 Z}^{A A}$ and $\sigma_{2 Z}^{A B}$ indicates, there is also a fair anount of tilting. The second part of the figure shows, on a condensed time scale, the rotation of the covariances along the exit coulomb trajectory. Finally, the third part shows the result of transforming to the external coordinate system $X Y Z$. This transformation is seen to have a substantial effect on the $x$-components; in fact ${ }^{A B}{ }_{X X}$ becomes negative.

The equiprobability contours of the fragment spin disiribution are elliosoids whose comon shape and orier.tation are detemined by the appropriatc covariances. In order to given a visual impression of the spin evolution we have included in Fig. 1 contours of the spiil distribution projected onto the $\times 2$ plane. One notes how the fairly peripheral collision ( $.440 \mathrm{~h}$ ) inhibits the bulld-up of negative spin modes so the distribution is very elongated. Furthermore, the smallness of the form factor prevents the distribution frr. aligning itself relative to the dinuclear axis. For $J=320 \mathrm{~s}$ the window grows wijer and the isotropic negative modes are more readily excited; the distribution also follows better the turning dinuclear axis. These features are even more apparent for $\mathrm{J}=100 \mathrm{~h}$.

\section{COMPARISON TO EXPER IMENT}

$\therefore$ present, our information about angular momentum in damped nuclear reactions arises from three types of observable: the mi:itiplicity of r-rays, the circular polarization of y-rays, and the angular correlation betwen the dirictiul. of motion of a reaction product and a sequential ejectile. In the present study we do not address the polarization data since they are yet fairly crude with respect to the dependence on energy loss.

The r-multiplicity data gives information about the distribution of the :0tal agnitude of fragment spin. When the spin fispersions are relatively smali, we nay obtain approxinzte expressicns for the spin nagnitura noments by expanding around the mean spin $\left\langle\bar{S}^{-f}\right.$. The leading order teras in the spin sagritute manents are the monents of the SDin distribution slong the reat ion normal. So minil the distribution hong the redtion mormal is proves in r-Tidiplicity experiments.

The renssion is preceted ty neutren evaboration which modifies the spin

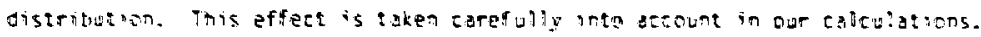


In Fig. 5 we show spin magnitude moments extracted from two sets of data on Y-multiplicities ${ }^{7,8}$ together with our calculated results. For the small TKEL, the calculated mean value of the $s p$ in is rising too steeply by a factor of two, relative to the experimental results. For the higher TKEL, the experimental values first reach a maximum and then decrease slightly towards the highest TKEL. The theoretical results reproduce these features and the maxinium has approximately the correct size, but it is reached at a too small TKEL, and the decrease for the highest TKEL is too pronounced.

The variances are in good agreement with the data. About $40 \%$ of the calculated variance is due to the correlation between the spins in the nuclei, especially the appreciable positive covariance ${ }^{A B} \mathrm{YY}$ along the reaction normal. The presence of this correlation explains in a natural way the rather large yariance of the sum of spin magnitudes seen in the r-multiplicity data.

The average spin magnitude provides information about the relative importance of different types of friction acting in the relative motion of the two nuclei during a collision. Our results indicate that the ratio between the radial and tangential friction, as obtained with the present implementation of the nuc leon exchange model, is too small.

For given impact parameter, the variance of the spin along the reaction normal is just given by the accumulated variance along the intrinsic y-direction. The variances along this direction increase rapidiy when the nuclei come into contact, and are dominated by the positive wriggling mode. However, the subsequent integration over impact parameters to obtain TKEL. gated distributions also contributes substantial variances and positive covariances so that the resulting dispersions become less sensitive to the specific model.
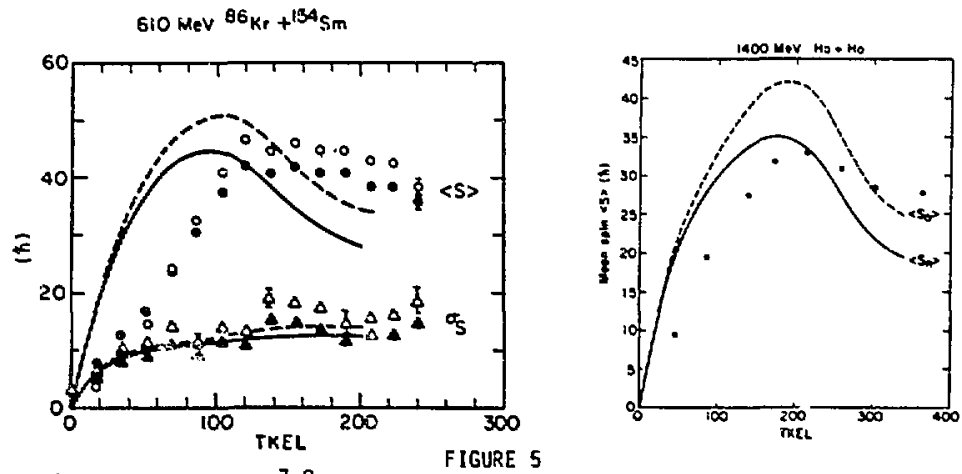

Calculated and measured 7,8 values of the mean spin magnitude and the associated covariance before (dashed) and after (solid) neutron evaporation. 
Angular correlation data gives information about the distribution of the spin directions. The moments of the directional distribution are the statistical spherical tensors. The tensor of rank two is the most important, giving the alignment along the reaction normal $P_{Y Y}=\left\langle\left(3 s_{Y}^{2}-s^{2}\right) / 2 S^{2}\right\rangle$ and the asymmetry between the in-plane components $P_{X Z}=\left\langle\left(S_{X}^{2}-s_{Z}^{2}\right) / s^{2}\right\rangle$. Thus, angular correlation experiments mainly probe the in-plane variances of the spin distribution in one nucleus, but only relative to the magnitude of the spin.

For a definite value of the spin magnitude in one of the reaction products, the terms of order zero and two of the angular correlation of sequential decay products emitted from that nucleus yield

$$
4 \pi \omega(\theta, \phi)=1+B_{2}(S) P_{Y Y}(S) P_{2}(\cos \theta)+\frac{3}{4} B_{2}(S) P_{X Z}(S) \sin ^{2} \theta \cos \phi .
$$

Here the coordinate system has the polar axis along the reaction normal and diagonalizes the in-plane spin variance tensor. $P_{2}$ is the second order Legendre polynomial and $B_{2}(S)$ is the second order angular distribution coefficient for the given $k$ ind of decay. For fission and for large spins $S>3 K_{0}$ we have approximately $8_{2}(S)=5\left(3 K_{0}^{2}-S^{2}\right) / 2 S^{2}$, where $k_{0}$ is the familiar parameter giving the dispersion of the distribution of $K$ quantum numbers of the saddle shape.

The tensor elements $P_{Y Y}(S)$ and $P_{X Z}(5)$ as well as the angular distribution coefficients and the fission probability vary with the spin magnitude $S$. In our actual calculations we include aiso fourth order terms and integrate over spin magnitude, taking into account competition between fission and neutron emission at all steps in a neutron decay cascade.

Figure 6 shows the experimental ${ }^{9}$ and calculated in-plane angular correlation for three different kinetic energy losses for each of the two reactions $\mathrm{Kr}+\mathrm{Bi}$ and $\mathrm{kr}+\mathrm{U}$. The calculation agrees well with ihe data regarding the position of the extrema of the in-plane angular correlation, along the principal axes of the spin variance tensor. [Only for TKEL $=170 \mathrm{MeV}$ in the $\mathrm{Kr}+$ Bi reaction do we get a discrepancy here, but this TKEL is in the calculation located at the upper edge of the energy loss distribution and is not focussed in scattering angle, so the discrepancy is not so serious.] The amplitude of the calculated variation is too small relative to the data by a factor of two. However, it should be kept in mind here that two other data sets for similar reactions with Bi or PD disagree with the data shown on Fig. 6: one $e^{10}$ displays in-plane anisotropies of approximately the same magnitude as calculated; and the other ${ }^{11}$ shows almost no in-plane variation. 


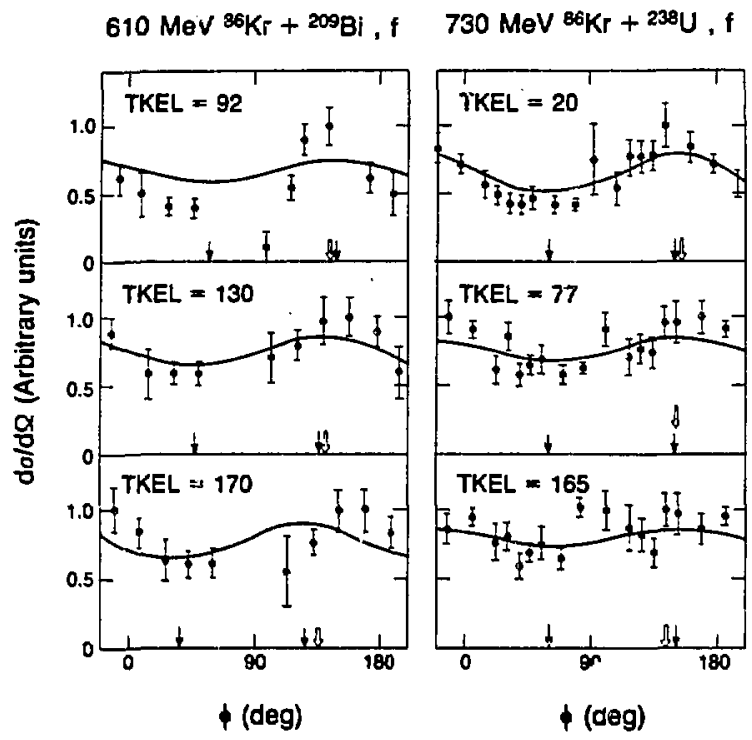

F IGURE 6

Calculated and measured ${ }^{9}$ angular correlation in the reaction plane for fission of the heavy nucleus produced in the reactions $610 \mathrm{MeV} 86 \mathrm{Kr}+209_{\mathrm{Bi}}$ and $730 \mathrm{MeV}$ $86_{k r}+238_{j} \phi=90^{\circ}$ corresponds to the beam axis. The open arrow for each TKE:- shows the target recoil direction in the lab-frame and the solid arrows point to the directions of principal axes of the in-plane components of the spin variance tensor.

The location of the maximum close to the average direction of the dinuclear axis during the reaction supports our result that the relaxation tiries for spin variances along the axis are longer than the reaction time and longer than the relaxation times for the spin variances perpendicular to the axis.

\section{CONCLUOING REMARKS}

The nucleon exchange transport model has addressed a variety of observables in damped nuclear reactions. The mass and charge distributions appear to be well described by the mode 1,12 and also the division of the excitation energy among the two fragments is in good accordance with data. ${ }^{13}$ He have refined the model considerably in order to achieve $s$ detailed description of the argular momentum accumulation, ${ }^{1}$ and have also deveioped the treatment of the subse- 
quent decay processes in order to calculate observed quantities. ${ }^{2}$ Spinrelated data obtained by different probes can then be confronted in a unified manner.

Our comparisons with data have yielded good agreement for a number of features, but the mean Spin magnitude appears to increase too rapidly with energy loss, suggesting the presence of an additional radial dissipation. Further studies are required to clarify whether this shortcoming is a consequence of the simple classical calculation of the transport coefficients or signals the action of a different damping mechanism.

on rather general grounds, the present model predicts specific correlations between the two fragment spins. The spin spin correlations contain valuable novel information about the reaction dynamics and their experimental determination offers the field an exciting new prospect.

\section{REFERENCES}

1) T. Obssing and J. Randrup, LBL-16825 (1983).

2) T. Obssing and J. Randrup, LBL-16826 (1983).

3) J. Randrup, Nucl, Phys. A327 (1979) 490.

4) J. Randrup, Nucl. Phys. A383 (1983) 468.

5) L.G. Moretto and R.P. Schmitt, Phys. Rev. C21 (1980) 204.

6) R.P. Schmitt and A.J. Pacheco, Nucl. Phys. A379 (1982) 313.

7) P.R. Christensen, O. Hansen, O. Nathan, F. Videbaek, H. Freiesleben, H., C. Briti and S.H. van der Werf, Nucl. Phys. A390 (1982) 336.

8) R.J. McDonald, A.J. Pacheco, G.J. Wozniak, H.H. Bolotin, L.G. Moretto, C. Schuck, S. Shih, R.M. Diamond and F.S. Stephens, Nucl. Phys. A373 (1982) 54.

9) P. Dyer, R.J. Puigh, R. Vandenbosch, T.D. Thomas, M.S. Zisman and L. Nunnelly, Nucl. Phys. A322 (1979) 205;

R.J. Puigh, F. Dyer, R. Vandenbosch, T.D. Thomas, L. Nunnelly and M.S. Zisman, Phys. Lett. 868 (1979) 24;

R. Vandenbosch (1983) priyate comunication.

10) D. von Harrach, P. Glassel, Y. Civelekoglu, R. Manner and H.J. Specht, Phys, Rev. Lett, 42 (1979) 1728.

11) C. Le Brun, J.F. Lecolley, F. Lefebures, M. L'Haridon, A. Osmont, J.P. Patry and J.C. Steckmeyer, in Proc. $X X$ int. Winter Meeting on Nucl. Phys., Bormio, Italy, January 1982 (University of Milano, 1982).

12) H.U. Schroder et al., Phys. Lett. 98B (1931) 355;

H.C. Britt et al., Phys. Rev. C26 (1982) 1999.

13) R. Vandenbosch, Horkshop on Nuclear Oynamics III, 5-9 March 1984, Copper Mountain, Colorado. 
This report was done with support from the Department of Energy. Any conciusions or opinions cxpressed in this report represent solely those of the author(s) and not necessarily those of The Regents of the University of California, the Lawrence Berkeley Laboratory or the Depariment of Encrgy.

Reference 10 a company or product name does not imply apprciai or rceonmenciation fi the product by the University of California or the U.S. Depariment of Encrgy to the exclusion of others that may be suitable. 